\title{
Terahertz Fourier Transform Spectrometer Based on a Low-Reflectivity Fabry-Perot Interferometer
}

\author{
Li-Jin Chen, Tzeng-Fu Kao, Hsu-Hao Chang, Ja-Yu Lu, and Chi-Kuang Sun \\ Graduate Institute of Electro-Optical Engineering, National Taiwan University, Taipei, 10617, Taiwan \\ Phone :886-2-23659703.FAX:886-2-23677467.E-mail : r92941014@ntu.edu.tw
}

\begin{abstract}
A simple THz Fourier transform spectrometer is demonstrated by using a lowreflectivity Fabry-Perot Interferometer. The emission spectra of a wavelength-tunable photonic transmitter excited by an optical coherent control system are determined by the spectrometer. (C2005 Optical Society of America

OCIS codes: (300.6300) Spectroscopy, Fourier transforms; (120.2230) Fabry-Perot;
\end{abstract}

\section{Introduction}

With the aid of ultrashort laser pulses, terahertz $(\mathrm{THz})$ technology has been developed with great progress. In past decades, many methods were proposed for generating and detecting the $\mathrm{THz}$ radiation and therefore many applications become realizable such as molecular recognition and material characterization. For promoting these applications, having a simple and reliable method for measuring the spectral characteristic of $\mathrm{THz}$ waves is as important as developing a high-power and high-efficiency $\mathrm{THz}$ emitter with tunable wavelength. For the spectral determination of the $\mathrm{THz}$ radiation, Fourier transform spectroscopy based on a Michelson Interferometer and $\mathrm{THz}$ time-domain spectroscopy (THz-TDS) based on photoconductive (PC) and electro-optic (EO) sampling are the most commonly used methods. However, the construction of the previous spectral characterization setup is complicated because of the invisibility of the THz waves and the long alignment distance. In addition, the setup of THz-TDS also has the disadvantage of immovability, not to mention that THz-TDS requires another intensive femtosecond optical pulse. Hence, a transmitted Fabry-Perot interferometer (FPI) which can be easily aligned with a visible light is proposed to perform the spectral analysis [1]. Taking the advantages of its compact size and movability, the Fabry Perot based spectrometer may be very attractive for many applications. But the complexity of spectrum reconstruction rises due to the interferences of multiple beams. In this paper, we propose a simple Fourier transform spectrometer based on a Fabry-Perot interferometer which has the merit of above methods. For eliminating the effect of multiple beam interference, a low-reflectivity Fabry-Perot spectrometer is adopted. In the case of low reflectivity, it will be shown that the interference is similar to the two-beam interference. So the spectrum can be reconstructed by taking a Fourier transform, which is just the same as the one-sided Fourier transform spectrometer. By utilizing this simple spectrometer, the emission spectra of a high-power and high-efficiency $\mathrm{THz}$ photonic transmitter $[2,3]$ excited by an optical coherent control system are determined [4]. We will show that the central wavelength of the $\mathrm{THz}$ radiation from the photonic transmitter is tunable by changing the delay of coherent control system. Combining this tunable radiation source with the simple spectrometer, a powerful $\mathrm{THz}$ spectral sensing system suitable for many applications is thus demonstrated.

\section{Theory and simulation results}

Consider an ideal FPI consists of two parallel reflecting surfaces. If the two surfaces are identical and each has an intensity reflection coefficient $R$, the power transmission through the device for different spacing is given by

$$
P_{\text {out }}(d)=\int P_{\text {in }}(f) T(d, f) d f,
$$

where

$$
\begin{aligned}
& T(d, f)=\frac{1}{1+H \sin ^{2}\left(\frac{2 \pi f n d \cos \theta}{c}\right)}, \\
& H=\frac{4 R}{(1-R)^{2}},
\end{aligned}
$$

and $P_{i n}$ is the power spectrum of the incident wave, $n$ is the refractive index of medium between two surfaces, $d$ is the spacing of the two surfaces, $c$ is the speed of light in vacuum, $\theta$ is the angle from normal incident, and $f$ is the frequency of radiation. Since the incident wave can be taken as a combination of various frequency components 


\section{QTuI2}

with different amplitudes, we may first consider a wave with single frequency $f_{0}$ for simplicity. For eliminating the effect of multiple-beam interference, we choose a pair of low reflective surfaces. When the intensity reflectivity $R$ is very low so that $H<<1$, equation (1) can be rewritten as follows.

$$
P_{\text {out }}(d)=\frac{1}{1+H \sin ^{2}\left(\frac{2 \pi f_{0} n d \cos \theta}{c}\right)} \approx 1-H \sin ^{2}\left(\frac{2 \pi f_{0} n d \cos \theta}{c}\right)=\left(1-\frac{H}{2}\right)+\frac{H}{2} \cos \left(\frac{4 \pi f_{0} n d \cos \theta}{c}\right)
$$

From (4) we can observe that the transmitted power will approximate to a one-sided cosine function with the amplitude of $((H / 2)$ and a de value of $(I-H / 2)$. Both amplitudes are affected by $H$ only. By removing the de value, we can retrieve a cosine waveform in the distance domain similar to the original waveform in the time domain. Comparing the distance domain signal with the time domain signal, the frequency of the former is $2 n \cos \theta / c$ times of the latter. Furthermore, since the cosine function is an even function which should be symmetrical about y-axis, we may mirror the one-sided signal about y-axis by letting $P_{\text {out }}(-d)=P_{\text {out }}(d)$. In this way, the spectrum can be reconstructed by taking a Fourier transform of $P_{\text {out }}$ and scaling the frequency axis by multiplying a value of $c / 2 n \cos$ $\theta$. So there is a perfect mapping between original frequency and reconstructed frequency. The algorithm we adopt here is the same as the ordinary Fourier transform spectroscopy. The resolution of this spectroscopy is limited by the maximum spacing FPI can separate.
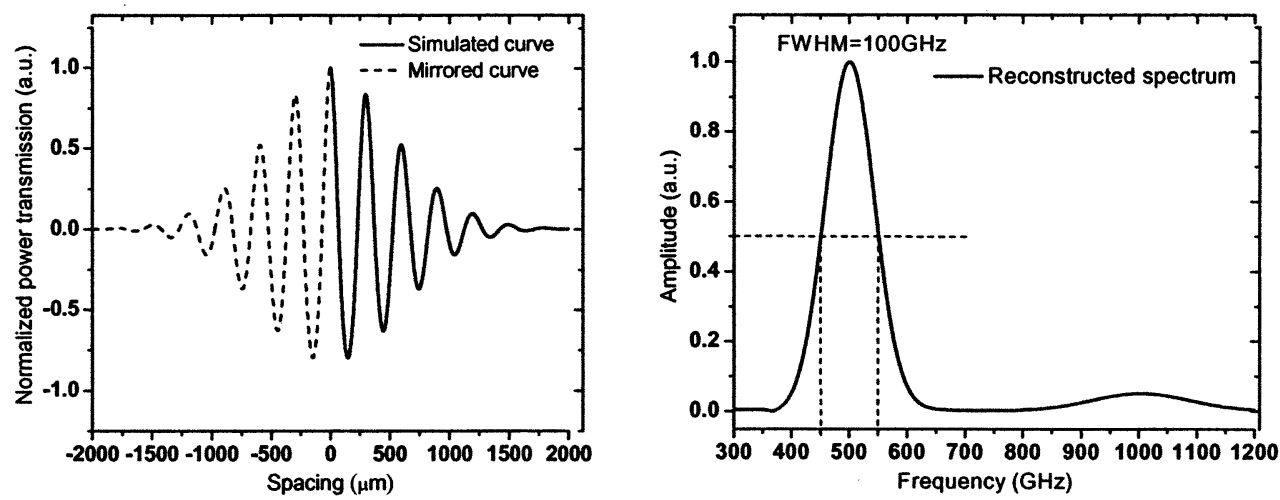

Fig. 1(a) Normalized power transmission of a Gaussian $\mathrm{THz}$ pulse with central frequency of $500 \mathrm{GHz}$ and FWHM of $100 \mathrm{GHz}$ for various spacing $d$ after removing the dc value (solid line) when $\mathrm{R}=10 \%$. The mirrored curve is shown in dashed line. (b) Reconstructed spectrum from the full trace plotted in Fig. 1(a) by taking the Fourier transform and scaling the frequency axis by multiplying $c \cos \theta / 2 n$.

Fig. 1(a) shows the simulated power transmission for various spacing $d$ after removing the dc value when $\mathrm{R}=10 \%$. The incident wave is assumed to be a Gaussian pulse with a FWHM of $100 \mathrm{GHz}$ and a central frequency of $500 \mathrm{GHz}$. The spectrum reconstructed by our algorithm is shown in Fig. 1(b). It can be observed that the reconstructed power spectrum restores the Gaussian peak located at $500 \mathrm{GHz}$ with a FWHM of $100 \mathrm{GHz}$, which is the same as the simulated incident wave. Unfortunately, the curve rises up slightly near $1 \mathrm{THz}$. This harmonic comes from the interference between the wave transmitted directly and the wave transmitted after two bounces. As the reflection coefficient $R$ increases, the harmonics become larger. Since the magnitude ratio of the second bounce to the first bounce wave is $R^{2}$, the effect of harmonic is reduced by using surfaces of low reflectivity. Therefore, it is important to keep R sufficiently small to avoid the influence of the harmonics for broadband $\mathrm{THz}$ analysis. On the other hand, with a know reflectivity value, it is possible to remove the undesired harmonic effect through a simple numerical algorithm.

\section{Experimental results}

Recently, we have demonstrated a edge-coupled membrane photonic transmitter based on a high bandwidthefficiency product MSM traveling-wave photodetector and a co-planar-waveguide fed slot antenna [2,3]. Because of its high power conversion efficiency, the photonic transmitter is suitable for many applications. For utilizing it as a narrow band THz emitter, an optical coherent control system is used to tune the central frequency of emission. This system has been previously demonstrated to have the capability to control the radiation frequency [4]. In order to further characterize the radiation spectra, the simple Fourier transform spectrometer based on a low-reflectivity Fabry-Perot interferometer is constructed. Fig. 2 shows the experiment setup of our $\mathrm{THz}$ excitation and 


\section{QTuI2}

measurement system. To generate the optical excitation pulses with a fixed beat frequency, an 100fs optical pulse with a central wavelength of $850 \mathrm{~nm}$ from a Ti:sapphire laser was first stretched to a linear chirp pulse of $8.7 \mathrm{ps}$ by a grating pair. Then, a Michelson interferometer was used to tune the beat frequency by controlling the relative delay of two arms. The radiated $\mathrm{THz}$ wave was collected by two parabolic mirrors and the Fourier transform spectrometer was located between them. The Fourier transform spectrometer was made of two sapphire backed metal meshes as the partial reflective mirrors. The reflectivity of each mirror at the $\mathrm{THz}$ wavelength is controlled to be $\sim 10 \%$. As the spacing between two mirrors changed, transmitted power detected by the bolometer changed too. Fig. 3 shows the measured $\mathrm{THz}$ power for different mirror spacing and the reconstructed radiation when the optical beating frequency was tuned to $460 \mathrm{GHz}$. The measured $\mathrm{THz}$ spectrum shows a peak radiation frequency $\sim 442 \mathrm{GHz}$ which is close to the excitation frequency of the photonic transmitter and the FWHM of the radiation is $\sim 50 \mathrm{GHz}$. The experimental result reveals that the radiation from the photonic transmitter is also quasi- $\mathrm{CW}$, following our expectation. This combined system with the simple $\mathrm{THz}$ spectrometer and the optically coherent controlled photonic transmitter thus provide us a reliable $\mathrm{THz}$ characterization tool for future spectroscopic applications.

In conclusion, we have demonstrated a simple Fourier transform spectrometer based on a low reflectivity FPI for spectral measurement. The spectrometer successfully characterizes the emission spectrum of an optically coherent controlled photonic transmitter. More characterization data on the photonic transmitter will be presented in the conference. Its limitation and comparison to other techniques will be also discussed.

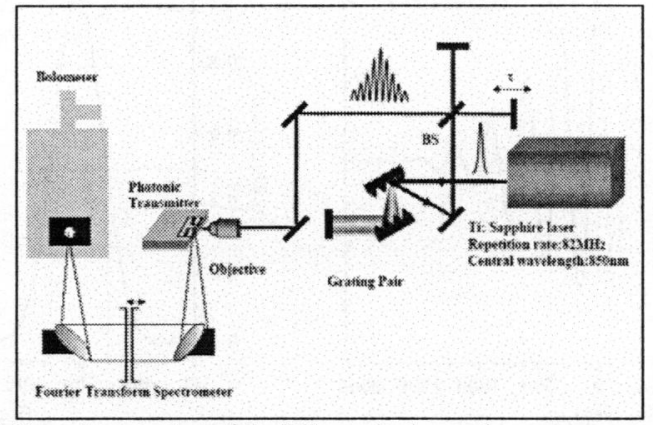

Fig. 2. Experiment setup of the THz excitation and measurement system.
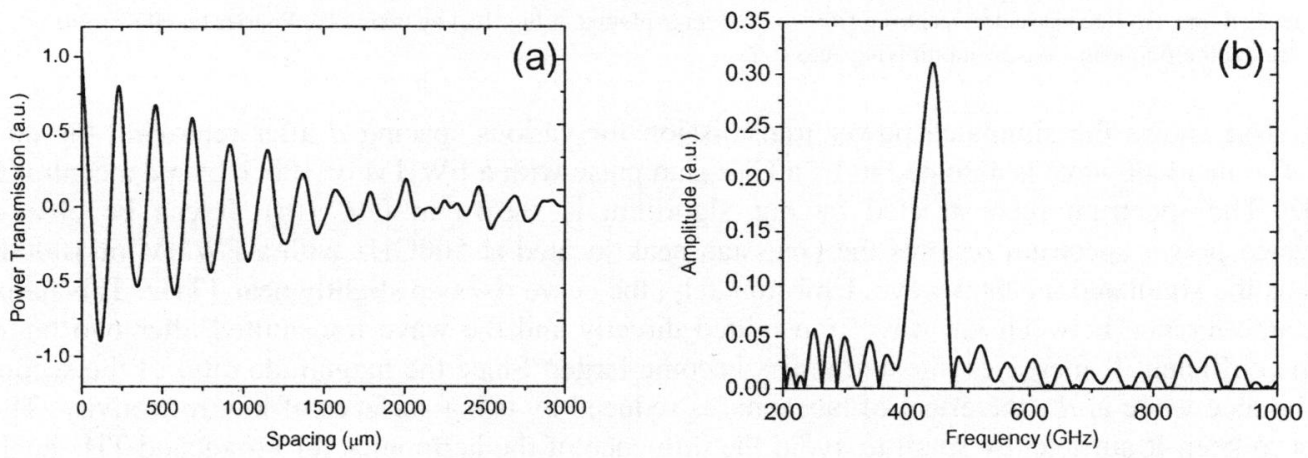

Fig.3. (a) Measured power transmission after removing the dc value when the excitation frequency is $460 \mathrm{GHz}$. (b) The reconstructed $\mathrm{THz}$ spectrum from (a)

\section{References}

[1] Y. Deng, et al., "Spectrum determination of terahertz sources using Fabry-Perot interferometer and bolometer detector," International Journal of Infrared and Millimeter Waves, 25, 215-228 (2004).

[2] J.-W. Shi, et al., "Edged-coupled membrane terahertz photonic transmitters based on metal-semiconductor-metal traveling-wave photodectors," Appl. Phys. Lett.., 81, 5108-5110 (2002).

[3] M.-C. Tien, et al., "Device saturation behavior of submillimeter-wave membrane photonic transmitters," IEEE Photonics Technology Letters, 16, 873-875 (2004)

[4] A. S. Weling, et al., "Novel sources and detectors for coherent tunable narrow-band terahertz radiation in free space," JOSA B, 13, $2783-2791$ (1996). 